\title{
Local conditions for the Pauli potential in order to yield self-consistent electron densities exhibiting proper atomic shell structure
}

Kati Finzel

\section{Linköping University Post Print}

\section{Tweet}

N.B.: When citing this work, cite the original article.

Original Publication:

Kati Finzel, Local conditions for the Pauli potential in order to yield self-consistent electron densities exhibiting proper atomic shell structure, 2016, Journal of Chemical Physics, (144), 3, 034108 .

http://dx.doi.org/10.1063/1.4940035

Copyright: American Institute of Physics (AIP)

http://www.aip.org/

Postprint available at: Linköping University Electronic Press

http://urn.kb.se/resolve?urn=urn:nbn:se:liu:diva-125312 


\section{AP $\mid \begin{aligned} & \text { The Journal of } \\ & \text { Chemical Physics }\end{aligned}$}

\section{Local conditions for the Pauli potential in order to yield self-consistent electron densities exhibiting proper atomic shell structure}

Kati Finzel

Citation: The Journal of Chemical Physics 144, 034108 (2016); doi: 10.1063/1.4940035

View online: http://dx.doi.org/10.1063/1.4940035

View Table of Contents: http://scitation.aip.org/content/aip/journal/jcp/144/3?ver=pdfcov

Published by the AIP Publishing

\section{Articles you may be interested in}

Robust acceleration of self consistent field calculations for density functional theory

J. Chem. Phys. 134, 134109 (2011); 10.1063/1.3574836

Beyond electronegativity and local hardness: Higher-order equalization criteria for determination of a groundstate electron density

J. Chem. Phys. 129, 054111 (2008); 10.1063/1.2957900

Self-consistent effective local potentials

J. Chem. Phys. 127, 084113 (2007); 10.1063/1.2768351

Local expansion of $\mathrm{N}$-representable one-particle density matrices yielding a prescribed electron density

J. Chem. Phys. 119, 8257 (2003); 10.1063/1.1611176

Electron distribution and capacitance-voltage profiles of multiple quantum well structure from self-consistent simulations

Appl. Phys. Lett. 70, 2987 (1997); 10.1063/1.118765

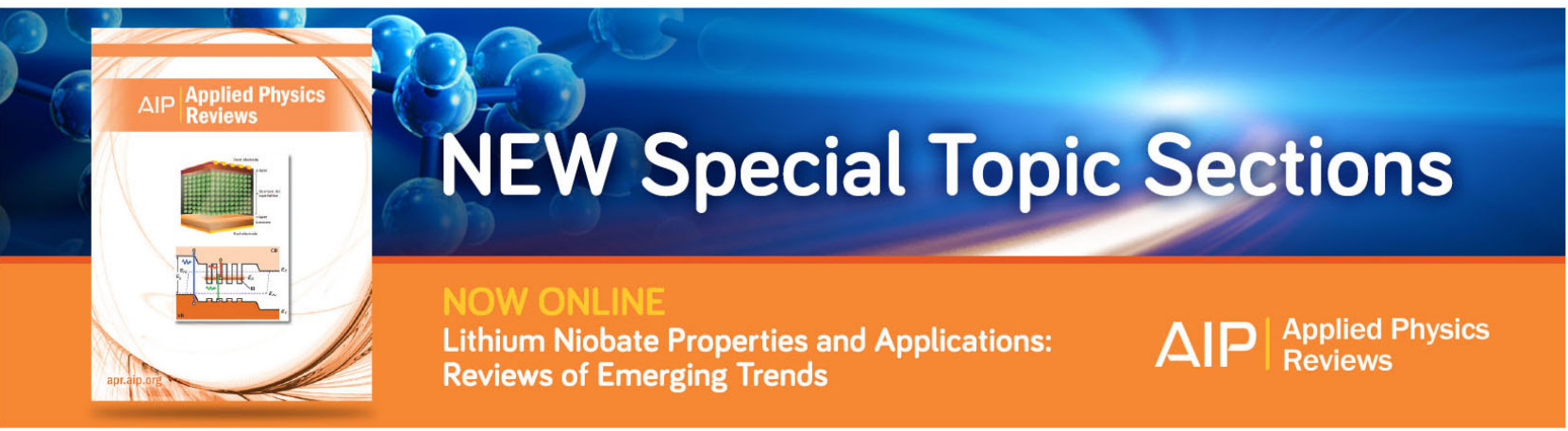




\title{
Local conditions for the Pauli potential in order to yield self-consistent electron densities exhibiting proper atomic shell structure
}

\author{
Kati Finzela) \\ Linköpings University, IFM Department of Physics, 58183 Linköping, Sweden
}

(Received 2 October 2015; accepted 4 January 2016; published online 20 January 2016)

\begin{abstract}
The local conditions for the Pauli potential that are necessary in order to yield self-consistent electron densities from orbital-free calculations are investigated for approximations that are expressed with the help of a local position variable. It is shown that those local conditions also apply when the Pauli potential is given in terms of the electron density. An explicit formula for the $\mathrm{Ne}$ atom is given, preserving the local conditions during the iterative procedure. The resulting orbital-free electron density exhibits proper shell structure behavior and is in close agreement with the Kohn-Sham electron density. This study demonstrates that it is possible to obtain self-consistent orbital-free electron densities with proper atomic shell structure from simple one-point approximations for the Pauli potential at local density level. (C) 2016 AIP Publishing LLC. [http://dx.doi.org/10.1063/1.4940035]
\end{abstract}

\section{INTRODUCTION}

The main challenge in orbital-free density functional theory $(\mathrm{OF}-\mathrm{DFT})^{1-5}$ is to find sufficiently accurate kinetic energy density functionals, leading to a reliable physical description of the system. Well known approximations for the kinetic energy as given by Thomas and Fermi, ${ }^{6,7}$ the von Weizsäcker functional, ${ }^{8}$ or the gradient expansion ${ }^{9,10}$ do not yield satisfactory results when applied with the Hohenberg-Kohn variational principle. ${ }^{11}$ Therefore, much effort has been undertaken in the direction of generalized gradient expansions, ${ }^{12-16}$ modified conjoint functionals (mcGGA) satisfying additional constraints, ${ }^{4,5}$ information-theory motivated functionals, ${ }^{17-19}$ and two-point functionals, usually based on response theory. ${ }^{1,20}$ However, progress seems to be hampered ${ }^{5}$ due to a lack of a systematic approach how to include the effect of the Pauli exclusion principle in an orbitalfree calculation. ${ }^{21}$ The problem how to obtain self-consistent electron densities exhibiting proper atomic shell structure has been an issue of long debate ${ }^{5,12,22}$ and is inevitably related to the problem how to impose proper $\mathrm{N}$-representability conditions on the functional ${ }^{23,24}$ in order to prevent a "variational catastrophe" in orbital-free implementations. ${ }^{23}$ The relationship between the Pauli potential and the atomic shell structure has already been studied a few years ago, ${ }^{25-27}$ still preserving interest in recent work on orbital-free density functional theory. ${ }^{28-30}$

Recently, a new group of so-called shell structure based functionals (SSB) has been proposed. ${ }^{31,32}$ SSB functionals approximate the Pauli kinetic energy based on the assumption that a proper representation of the atomic shell structure in real space $^{33-43}$ is mandatory for the performance of the functional itself. It has been shown that imposing conditions for proper atomic shell structure behavior on the Pauli potential directly leads to approximations that are able to produce self-consistent electron densities exhibiting proper atomic shell structure ${ }^{32}$ for

\footnotetext{
a)kati.finzel@liu.se
}

the radial density. Those conditions have been imposed with the help of a local position coordinate. This study deepens the investigations how to impose local constraints on the Pauli potential in order to yield proper self-consistent electron densities and additionally presents a route how to fulfill those local conditions for the Pauli potential in terms of the electron density.

\section{THEORY}

The main challenge in orbital-free DFT is to solve the Euler equation ${ }^{11}$

$$
0=\frac{\delta E}{\delta \rho}-\mu
$$

under the constraint, $0=\mu\left(\int \rho(\vec{r}) \mathrm{d} \vec{r}-N\right)$, that the electron density $\rho$ stays normalized to the number of electrons $N$ in the system. Neglecting correlation effects, the electronic energy $E$ for a closed shell atom of nuclear charge $Z$ is given by

$$
E[\rho]=T_{W}[\rho]+T_{\mathrm{P}}[\rho]+E_{Z}[\rho]+E_{H}[\rho]+E_{X}[\rho] .
$$

In the above equation, the kinetic energy $T=T_{\mathrm{W}}+T_{\mathrm{P}}$ has been divided into the von Weizsäcker term

$$
T_{\mathrm{W}}[\rho]=\int \frac{1}{8} \frac{(\nabla \rho(\vec{r}))^{2}}{\rho(\vec{r})} \mathrm{d} \vec{r}
$$

and the Pauli term $T_{P}$, which is defined as the remainder ${ }^{21,44}$ between the kinetic energy of the system and the energy of a bosonic system in its ground state, having the same density like the fermionic system. In terms of orbitals, the Pauli kinetic energy is expressed as

$$
T_{\mathrm{P}}=\int\left(\frac{1}{2} \sum_{i}^{\text {occ }}\left(\nabla \phi_{i}(\vec{r})\right)^{2}-\frac{1}{8} \frac{(\nabla \rho(\vec{r}))^{2}}{\rho(\vec{r})}\right) \mathrm{d} \vec{r},
$$

whereby the sum runs over all occupied spin orbitals. The Coulomb part $V$ of the total energy $(E=T+V)$, cf. Eq. (2), 
is given as the sum of the electron-nuclear attraction

$$
E_{Z}[\rho]=-Z \int \frac{\rho(\vec{r})}{\vec{r}} \mathrm{~d} \vec{r},
$$

the Hartree term, accounting for the electron-electron repulsion

$$
E_{H}[\rho]=\frac{1}{2} \iint \frac{\rho(\vec{r}) \rho\left(\vec{r}^{\prime}\right)}{\left|\vec{r}-\vec{r}^{\prime}\right|} \mathrm{d} \vec{r} \mathrm{~d} \vec{r}^{\prime}
$$

and the exchange term

$$
E_{X}[\rho]=-C_{X} \int \rho^{4 / 3}(\vec{r}) \mathrm{d} \vec{r},
$$

whereby $C_{X}=\frac{3}{4 \pi}\left(3 \pi^{2}\right)^{1 / 3}$.

With the transformation $y=r \sqrt{\rho}$ the Euler equation, cf. Eq. (1), for a spherical system finally results in

$$
0=-\frac{\partial^{2} y}{\partial r^{2}}+q(r) y
$$

with

$$
\begin{aligned}
q(r)= & 2 v_{\mathrm{P}}-2 \frac{Z}{r}-2 \mu \\
& +2\left[\frac{N}{r}-\frac{1}{r} 4 \pi \int_{r}^{\infty} y\left(r^{\prime}\right)^{2} \mathrm{~d} r^{\prime}+4 \pi \int_{r}^{\infty} \frac{y\left(r^{\prime}\right)^{2}}{r^{\prime}} \mathrm{d} r^{\prime}\right] \\
& -2\left(\frac{3}{\pi}\right)^{1 / 3}\left(\frac{y}{r}\right)^{2 / 3},
\end{aligned}
$$

whereby the Pauli potential

$$
v_{\mathrm{P}}=\frac{\delta T_{\mathrm{P}}}{\delta \rho}
$$

remains the only term which is needed to be approximated in terms of the electron density.

\section{COMPUTATIONAL DETAILS}

In order to assure convergence of the iterative process, the so-called $\lambda$-method ${ }^{32}$ is chosen for approximately solving the integro-differential equation, cf. Eq. (8). Adding $\lambda$ times the requirement $y=y$ to the original equation, the new differential equation to solve is

$$
\lambda=-\frac{\partial^{2} y}{\partial r^{2}}+\left[q(r)+\frac{\lambda}{\tilde{y}}\right] y,
$$

whereby the terms in square brackets are evaluated from the solution of the previous run, noted by $\tilde{y}$. In the above equation, the differential is approximated by a finite difference and the corresponding set of algebraic equations is solved via Gaussian elimination. A detailed description of the method can be found elsewhere. ${ }^{32}$

For a fully converged cycle $\tilde{y}=y$, so that the additional terms in Eq. (11) cancel, and $y$ is also the solution to the original differential equation, cf. Eq. (8). In practice, convergence will be reached up to a certain threshold and the results of two consecutive runs will slightly differ from each other. This implies that the solution obtained from Eq. (11) is only an approximation to the solution of the original Euler equation (8), whereby the difference between the two functions manifests especially in the tail region, where $\tilde{y}$ is small and increases with increasing $\lambda$, cf. Eq. (11). The quality of the approximative solution $y$ can be monitored by tightening the convergence criterion

$$
d_{2}(\tilde{y}, y)=\sqrt{\int(\tilde{y}-y)^{2} \mathrm{~d} \vec{r}}
$$

between two consecutive runs. The present calculations have been obtained with $d_{2}(\tilde{y}, y)=10^{-3}$ a.u. and $\lambda=15$ for all examined Ansätze of the Pauli potential. Calculations have been performed on an equidistant grid of $10^{-4}$ a.u. step size. The Lagrange multiplier $\mu$ was chosen such that

$$
\int \rho(\mu ; \vec{r}) \mathrm{d} \vec{r}-N \leq 0.001
$$

in order to keep the solutions normalized. The orbital-free results are compared with Kohn-Sham (KS) calculations performed with the ADF program ${ }^{45}$ using pure LDA exchange (Xonly).

\section{LOCAL CONDITIONS FOR THE PAULI POTENTIAL IN TERMS OF A LOCAL POSITION VARIABLE}

A simple approximation for the Pauli potential yielding self-consistent electron densities exhibiting proper atomic shell structure was given in Ref. 32. For atomic systems in their ground state, the so-called SSB-step potential is a stepwise function, whereby the number of steps equals the row of the element in the periodic table and the height of the steps correlates with the difference between the square of the effective nuclear charges ${ }^{46,47}$ for two successive atomic shells.

The SSB-step potential for the $\mathrm{Ne}$ atom as well as the corresponding radial density (scaled by factor 4 for better visibility) are schematically drawn in Fig. 1, immediately revealing the local conditions for the Pauli potential that are necessary in order to yield electron densities with proper atomic shell structure. As can be seen from the figure, $v_{\mathrm{P}}(r)$ must be constructed in such a way that the resulting $q(r)$, cf. Eq. (8), is positive in the region where the function $y(r)$ shall exhibit a minimum.

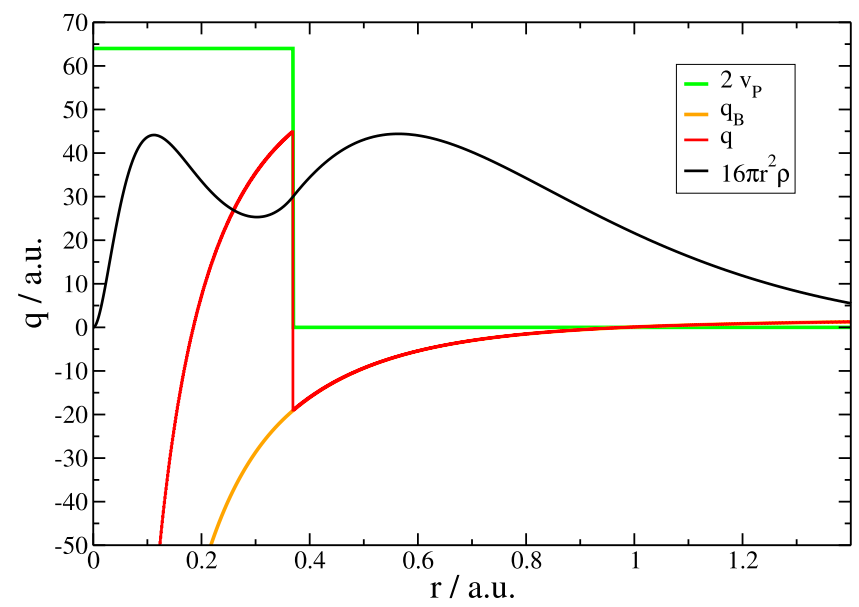

FIG. 1. Schematic draft of the influence of the Pauli potential $v_{\mathrm{P}}$ on the atomic shell structure of the radial density. The function $q$ is given by Eq. (8) and $q_{\mathrm{B}}=q-2 v_{\mathrm{P}}$ 
The remaining bosonic like term $q_{\mathrm{B}}=q-2 v_{\mathrm{P}}$ is negative for a large region around the atomic nucleus. Positive values for $q_{\mathrm{B}}$ are only found at large distances. Since $q_{\mathrm{B}}$ exhibits only one root, the corresponding $y_{\mathrm{B}}$, originating from the solution of Eq. (8) with $v_{\mathrm{P}}=0$, will display only one maximum. In order to display the characteristics of the atomic shell structure, meaning that the number of maxima $n$ of the radial density equals the row of the periodic table for this element, the function $q(r)$ has to exhibit $(2 n-1)$ roots. To achieve this, $v_{\mathrm{P}}$ has to be greater than $q_{\mathrm{B}}$ for position values $r$ lying between an odd and an even root of $q$. Those roots $z_{i}$, if they exist will of course be the turning points of the function $y$. Those local conditions apply to all atoms in the periodic table, and are likely to be extendable to molecules and solids. However, the local conditions are non-universal in the sense that they cannot be applied to non-atomistic model systems like the homogeneous electron gas.

The above mentioned local conditions have to be fulfilled in each step of the iterative process for the self-consistent electron densities. Fig. 2 displays the SSB-step potential (multiplied by factor 2) for the $\mathrm{Ne}$ atom as well as the function $-q_{\mathrm{B}}$ for each iteration cycle. Remember that $v_{\mathrm{P}}$ is kept fixed during the self-consistent iteration procedure. ${ }^{32}$ As can be seen from the data, $2 v_{\mathrm{P}}>q_{\mathrm{B}}$ for $\left.r=\right] z_{0}, z_{1}$ [ during the complete iteration process. This is the reason why the atomic shell structure is introduced (the starting point is a single exponential function) and maintained during the variational process.

On the other side, it seems worthy to investigate further approximations to the Pauli potential which are locally closer to its KS counterpart than is the SSB-step potential. It is found that

$$
v_{\mathrm{P}}(r)=\frac{a r^{\alpha}+b}{c r^{\beta}+1}
$$

with positive $a, b, c, \alpha, \beta$ and $\beta>\alpha$ yields a good description of the Pauli potential for second row elements. In the above expression, the parameter $b$ correlates with the energy difference between the first and the second shell. Since energy eigenvalues are not part of an orbital-free approach, an additional model for the $b$ value is needed. As a first

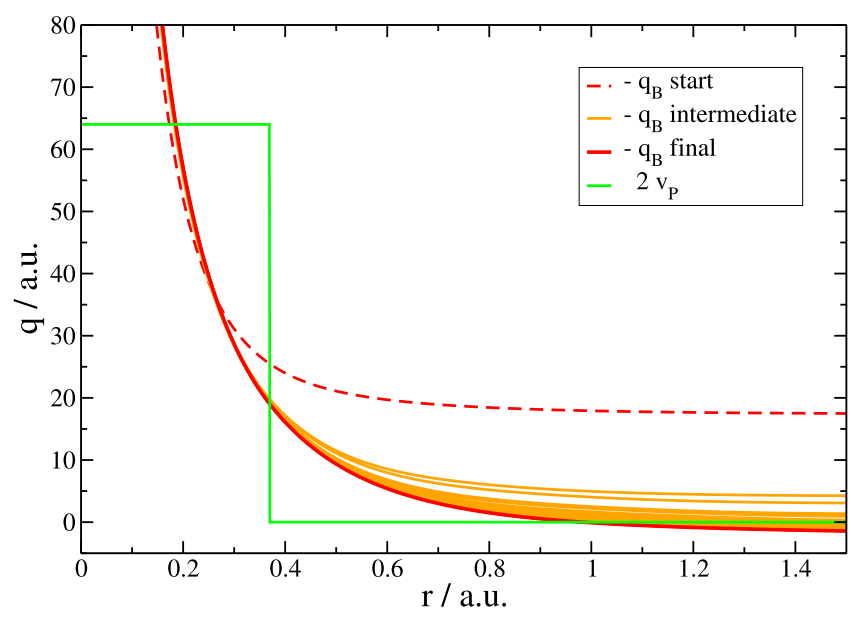

FIG. 2. SSB-step potential and $q_{\mathrm{B}}$ for the Ne atom during the self-consistent iteration process. estimate, $b$ can be evaluated from the energy eigenvalues of the one-electron hydrogen like Schrödinger-equation. Next, $a$ and $c$ shall be chosen such that the maximum of the Pauli potential equals the ideal shell boundary $r_{1}^{35}$ between first and second atomic shell. With $a=\kappa c$, the $c$ value for which the maximum of $v_{\mathrm{P}}$ lies at $r_{1}$ is given by

$$
c=\frac{b \beta}{r_{1}^{\alpha} \kappa(\alpha-\beta)}-\frac{\alpha}{r_{1}^{\beta}(\alpha-\beta)} .
$$

For $\beta>\alpha$, the $c$ value is positive if $\kappa$ is chosen above $\kappa_{0}$,

$$
\kappa_{0}=r_{1}^{\beta-\alpha} \frac{b \beta}{\alpha} .
$$

The value for the first shell boundary for all elements from $\mathrm{Li}$ to $\mathrm{Xe}$ is in good approximation given by $r_{1} \approx \ln (Z /(Z-2)){ }^{48}$ A detailed investigation of the ideal atomic shell structure and its application in the design of kinetic energy density functionals is given elsewhere. ${ }^{48}$

Table I compiles the chosen parameter values for the investigated approximations according to Eq. (14). The first group consists of functions with $\alpha=2$ and $\beta=4$. The corresponding potentials are drawn in Fig. 3. The Pauli potential calculated from KS orbitals as well as the SSB-step potential are given for comparison. In Ansatz 1-4, the value for $b=37.5$ has been calculated from the difference between

TABLE I. Parameter values for the different Ansätze according to Eq. (14).

\begin{tabular}{lccccrrr}
\hline \hline Ansatz & $r_{\max } /$ a.u. & $\alpha$ & $\beta$ & $\kappa /$ a.u. & $a /$ a.u. & $b /$ a.u. & $c /$ a.u. \\
\hline 1 & 0.223 & 2 & 4 & 3.8 & 26.43 & 37.5 & 6.95 \\
2 & 0.223 & 2 & 4 & 4.0 & 107.09 & 37.5 & 26.77 \\
3 & 0.223 & 2 & 4 & 4.5 & 308.76 & 37.5 & 68.61 \\
4 & 0.223 & 2 & 4 & 5.0 & 510.43 & 37.5 & 102.09 \\
5 & 0.223 & 2 & 4 & 4.0 & 207.51 & 35.0 & 51.88 \\
6 & 0.223 & 2 & 4 & 4.0 & 408.34 & 30.0 & 102.09 \\
7 & 0.223 & 2 & 4 & 4.0 & 448.51 & 29.0 & 112.13 \\
8 & 0.223 & 2 & 4 & 4.0 & 609.17 & 25.0 & 152.29 \\
9 & 0.223 & 4 & 6 & 3.0 & 13511.12 & 29.0 & 4503.71 \\
10 & 0.223 & 4 & 6 & 2.5 & 5410.95 & 29.0 & 2164.38 \\
11 & 0.223 & 4 & 8 & 0.25 & 17275.91 & 29.0 & 69103.66 \\
12 & 0.223 & 4 & 8 & 0.20 & 9142.08 & 29.0 & 45710.40 \\
\hline \hline
\end{tabular}

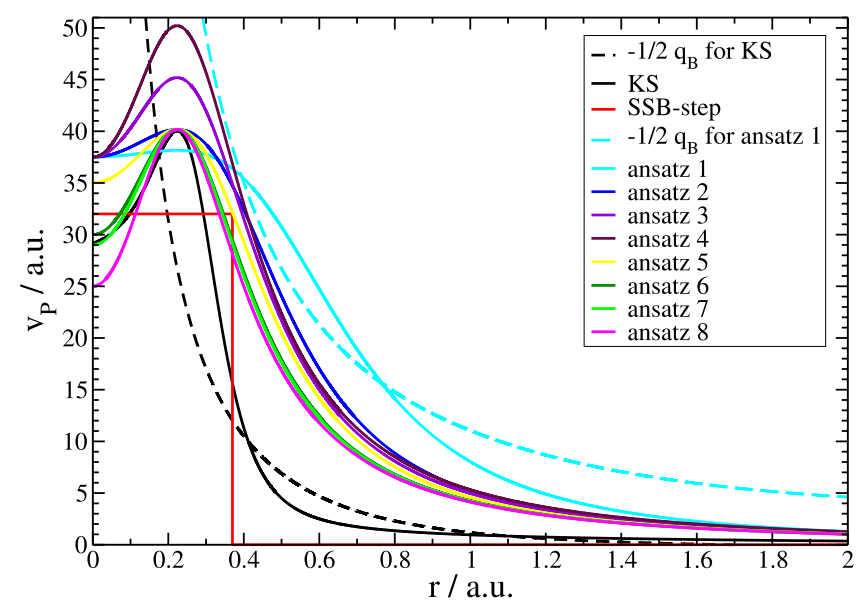

FIG. 3. Pauli potential as given by Eq. (14) for parameter values given in Table I. 


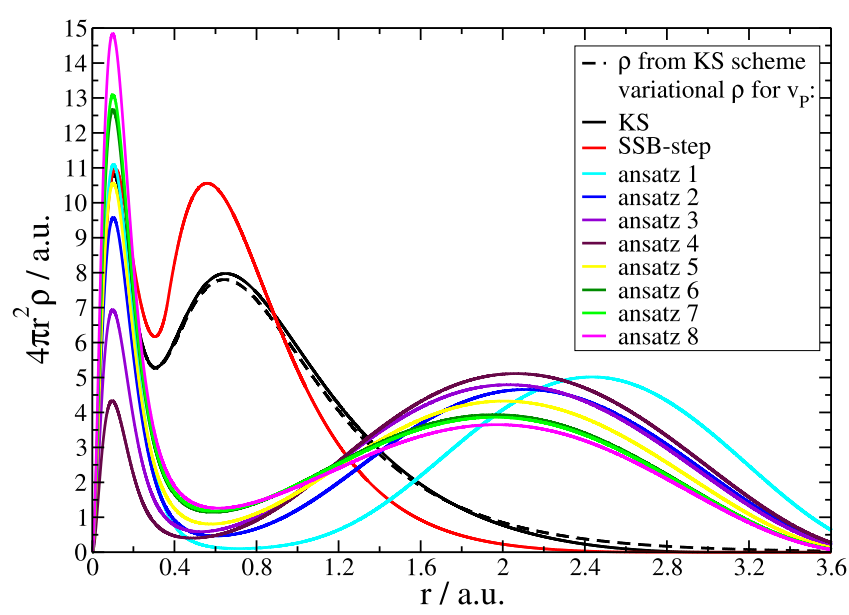

FIG. 4. Self-consistent orbital-free radial electron densities obtained from Ansätze for the Pauli potential shown in Fig. 3.

the first and the second energy eigenvalue of the one-electron Schrödinger equation with $Z=10$, yielding a rough estimate for the Pauli potential close to the nucleus. For the subsequent Ansätze, the $b$ values have been successively decreased in order to obtain a better agreement with the KS Pauli potential. All approximative Pauli potentials have been chosen in such a way that the maximal value corresponds to the shell boundary $r_{1}=0.223$ a.u. Fig. 3 also contains the function $-1 / 2 q_{\mathrm{B}}$ for the KS calculation as well as for Ansatz 1 (the most widespread Ansatz), revealing that all resulting radial electron densities will display the proper number of maxima, since $2 v_{\mathrm{P}}>q_{\mathrm{B}}$ for $r=] z_{0}, z_{1}[$. The data for the resulting self-consistent electron densities are shown in Fig. 4. All Ansätze yield the proper number of extrema for the radial electron density. However, the overall correspondence between those densities and the $\mathrm{KS}$ density is unsatisfactory. This is due to the wrong decaying behavior of the Ansätze in the second shell. For all Ansätze with $\alpha=2, \beta=4$, the decay in the second shell is much too slow. Consequently, the second maximum of the radial density is shifted outwards, whereby the shift is most pronounced for Ansatz 1 having the largest Pauli potential in the second shell region. Remark that decreasing the Pauli potential in the second shell does also lower the electronic population in the first shell, compare, e.g., the data of Ansatz 1 shown in light blue with the data for Ansatz 2 shown in dark blue. In general, lower values for the Pauli potential in the corresponding shell region lead to higher values of the radial density for this shell, compare especially the data for Ansätze 5-8.

Interestingly, the radial density stemming from the SSBstep potential is in best agreement with the KS density. Thus, an underestimation of the Pauli potential in the second shell seems to affect the position of the second shell maximum much less than an overestimate. Although, in case of the SSB-step potential the maximum of the corresponding radial density is too high compared to the $\mathrm{KS}$ data, due to that underestimation.

The above analyses of the electron densities obtained with the Ansätze 1-8 for the Pauli potential show that a sufficiently rapid decay of the Pauli potential in the second shell is mandatory in order to yield electron densities from the variational procedure that are reasonably close to the KS density. A rapid decay of the Pauli potential given by Eq. (14) can be enforced by choosing $\beta>4$. The examined Ansätze 9-12 are shown in Fig. 5 and the corresponding parameters are listed in Table I.

Compared to the Pauli potential from KS orbitals the decay of Ansätze 9 and 10 is still not sufficiently steep. Consequently, the maximum for the second shell in the resulting radial electron densities, shown in Fig. 6, is still shifted towards higher radial values. Note that the height of the maximal value of the Pauli potential has a significant influence on the atomic shell structure of the resulting electron density. Approximations to the Pauli potential, for which the kink at the first shell boundary is not sufficiently pronounced, will fail to produce electron densities with proper atomic shell structure, compare the results for Ansätze 9 and 10. By choosing $\alpha=4, \beta=8$ the rapid decay of the Pauli potential in the second shell region can be reproduced sufficiently well, see Ansätze 11 and 12 in Fig. 5. Those Ansätze yield the best overall agreement between the orbital-free electron density and the KS electron density. Notice that an overestimate of the maximal value of the Pauli potential at the shell boundary leads to smaller electron density values in the first shell region compared to the KS solution.

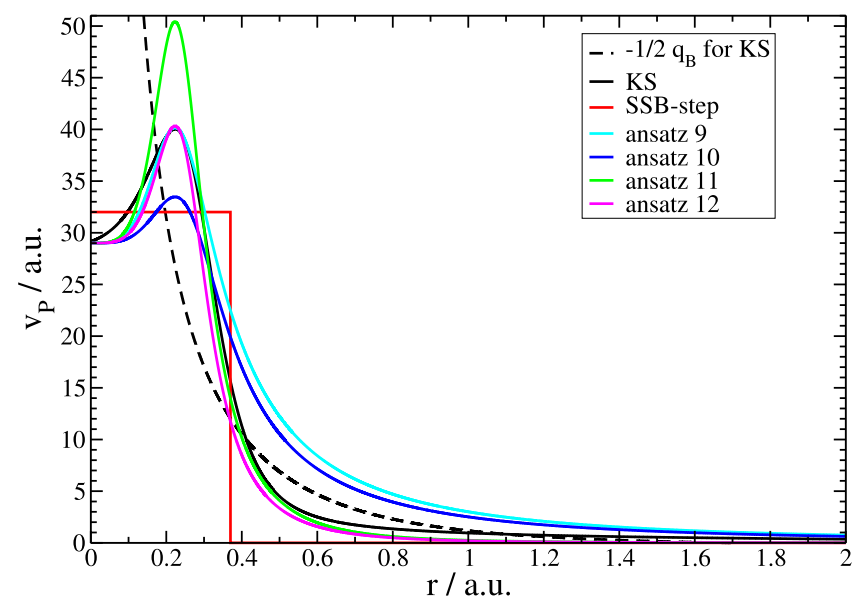

FIG. 5. Ansätze for the Pauli potential, cf. Eq. (14), with rapid decay in the second shell region. Parameter values are given in Table I.

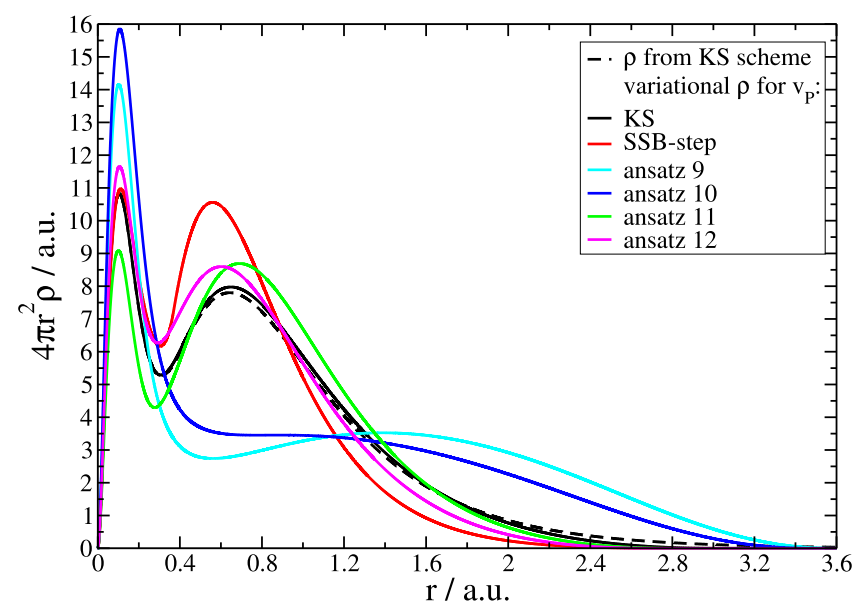

FIG. 6. Self-consistent orbital-free radial electron densities obtained from Ansätze for the Pauli potential shown in Fig. 5. 
Finally, the local conditions for the Pauli potential in order to yield self-consistent electron densities exhibiting proper atomic shell structure can be stated as: It is mandatory that the resulting function $q(r)$ exhibits $(2 n-1)$ roots. For Pauli potentials expressed with the help of a local position variable this requirement can be achieved by functions displaying $n$ sharp maxima at the ideal shell boundaries. It is further desirable that those functions have a sufficiently rapid decay in the outermost shell region.

\section{LOCAL CONDITIONS FOR THE PAULI POTENTIAL IN TERMS OF THE ELECTRON DENSITY}

In the section titled "Local conditions for the Pauli potential in terms of a local position variable," it was shown how to design an approximate Pauli potential leading to radial electron densities exhibiting proper atomic shell structure with the help of a local position variable. This section deals with the problem how to approximate the Pauli potential in terms of the electron density still preserving the local conditions from the section titled "Local conditions for the Pauli potential in terms of a local position variable."

Taking the SSB-step potential as an illustrative example, the Pauli potential as well as the function $-1 / 2 q_{\mathrm{B}}$ have been plotted as a function of the electron density during the iterative process. The data are shown in Fig. 7. Since in Eq. (8) the term $q$ can be either seen as a function of $r$ or as a function of $\rho$, the local conditions for the Pauli potential (expressed in terms of $\rho$ ) yielding electron density with proper atomic shell structure are the same as in the section titled "Local conditions for the Pauli potential in terms of a local position variable." The function $q(\rho)$ must exhibit $(2 n-1)$ roots with $n$ being the row of the element in the periodic table. The region of interest leading to the minimum in the radial distribution function for the $\mathrm{Ne}$ atom is located around $\rho=10$ a.u., see Fig. 7. For the SSB-step potential $v_{\mathrm{P}}(\rho)>-1 / 2 q_{\mathrm{B}}(\rho)$ for $\left.r=\right] z_{0}, z_{1}[$ in each step during the iterative process. The challenge is to find such an Ansatz for the Pauli potential in terms of the electron density that displays the proper number of roots for $q$ for all reasonable electron densities during the variational

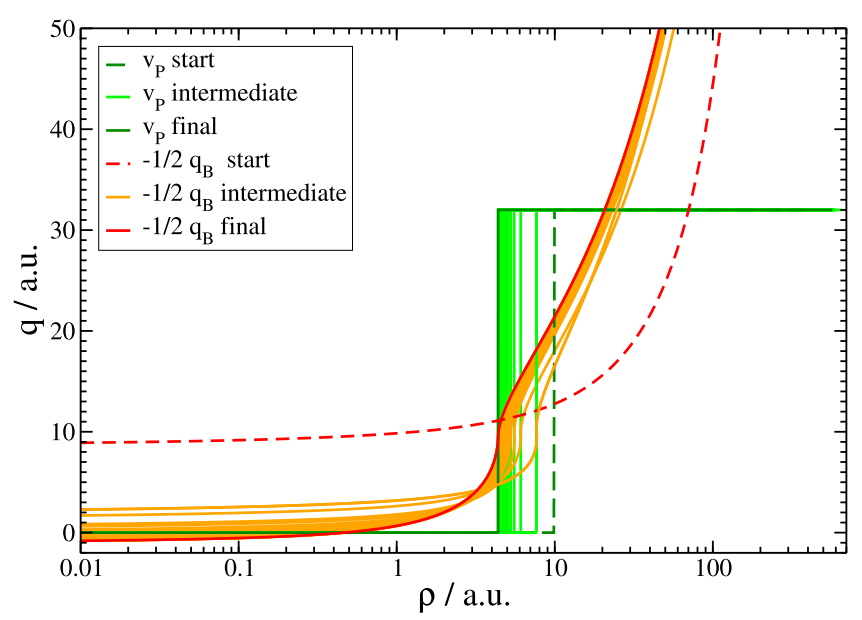

FIG. 7. SSB-step potential during iterative process as a function of the electron density.

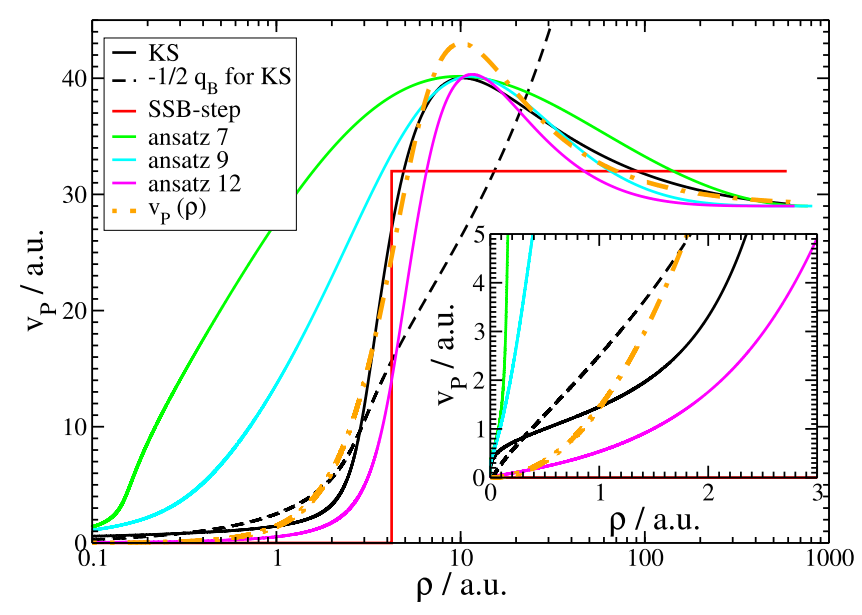

FIG. 8. Pauli potential for various Ansätze as a function of the electron density.

procedure. Of course one can always imagine Gedanken densities ${ }^{49}$ that will not lead to the desired behavior of $q$. For this reason, a given approximation for the Pauli potential based on the assumption of the necessity to reveal the atomic shell structure is expected to be restricted to applications on atomistic systems, like atoms, molecules, and solids see also the discussion in the section titled "Local conditions for the Pauli potential in terms of a local position variable."

In order to find a suitable approximation for the Pauli potential, the most promising Ansätze from the section titled "Local conditions for the Pauli potential in terms of a local position variable" are investigated in terms of the electron density. The data are compiled in Fig. 8, showing Ansätze 7, 9, and 12 as well as the Pauli potential evaluated from KS orbitals. The function $-1 / 2 q_{\mathrm{B}}$ for the KS calculation is also given for a rough estimate of the roots of $q$ for the examined Ansätze. The region located around $\rho \approx 10$ a.u., where $v_{\mathrm{P}}(\rho)>-1 / 2 q_{\mathrm{B}}(\rho)$, is the region where the radial electron density exhibits its minimum. Ansätze 7 and 9 exhibit proper behavior for electron densities close to zero, since $\beta-\alpha=2$. However, with $\beta-\alpha=2$ the rapid decay of the Pauli potential in the second shell cannot be modeled satisfactorily. Instead, with $\beta-\alpha=4$, see data for Ansatz 12 , the overall correspondence with the KS Pauli potential is much better. Note that the SSB-step potential which is zero in the second shell also yields electron densities in close agreement with the KS electron density. Therefore, the aim is to construct such an approximation to the Pauli potential in terms of $\rho$, with sufficiently small values for low electron densities and the proper limiting value at the nucleus (high density limit). The maximum of $v_{\mathrm{P}}(\rho)$ shall be located at the electron density for the ideal shell boundary between first and second shell.

Reasonable approximations for the Pauli potential in terms of the electron density can be generated by the following Ansatz:

$$
v_{\mathrm{P}}(\rho)=\frac{k \rho^{\gamma}-l}{m \rho^{\epsilon}+1}+l
$$

with positive $k, l, m, \gamma, \epsilon$ and $\epsilon>\gamma$. By comparison with Eq. (14), the parameter $l$ can be identified as $b$, since it 


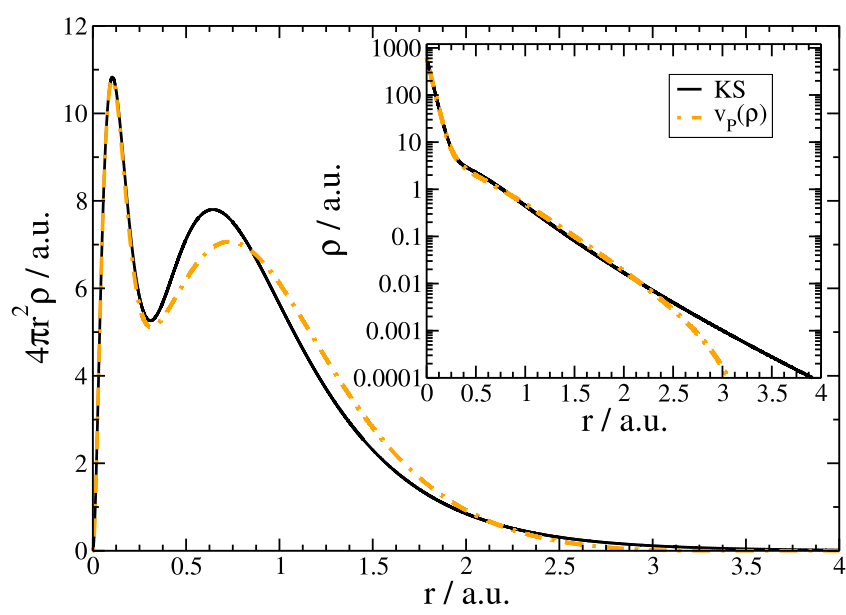

FIG. 9. Self-consistent electron density obtained from orbital-free calculation with the Pauli potential given in terms of the electron density.

represents the limiting value for the Pauli potential at the nucleus. As a first estimate that value can be evaluated from the eigenvalues of the one-electron Schrödinger equation. Next, $k$ and $m$ must be chosen such that the maximum of the Pauli potential equals the electron density at the ideal shell boundary. With $k=\eta m$, the $m$ value for which the maximum of the Pauli potential lies at $\rho_{1}$ is given by

$$
m=\frac{-\epsilon l}{\rho_{1}^{\gamma} \eta(\gamma-\epsilon)}-\frac{\gamma}{\rho_{1}^{\epsilon}(\gamma-\epsilon)},
$$

whereby $\rho_{1}$ is the electron density at the first shell boundary. For the $\mathrm{Ne}$ atom that value is $\rho_{1} \approx 10$ a.u. (for the KS electron density). Fulfilling the above mentioned requirements, a suitable approximation for the Pauli potential of the $\mathrm{Ne}$ atom is obtained from Eq. (17) by choosing $\gamma=2, \epsilon=3$, $k=1.27$ a.u., $l=29.0$ a.u., $m=0.006$ a.u., shown by the dashed-dotted orange line in Fig. 8.

With that explicit expression for the Pauli potential given in terms of $\rho$ the Euler equation has been solved for the $\mathrm{Ne}$ atom. The resulting orbital-free self-consistent electron density is shown in Fig. 9. As can be seen the orbital-free radial electron density displays the proper number of maxima. Additionally, the values themselves are in close agreement with the corresponding results from KS calculation, compare also the logarithmic plot of the electron densities shown in the inset of Fig. 9. This example demonstrates that it is possible to obtain self-consistent electron densities with proper atomic shell structure from orbital-free calculations with an explicit expression for the Pauli potential given in terms of the electron density.

\section{DISCUSSION}

The reader might have noticed two issues that are inherently related to the current approach. The first point is that the potential and the corresponding kinetic energy functional are non-universal, and that its parameterization depends on the chosen atom. The second issue is that the proposed approximation for the Pauli potential and the corresponding kinetic energy density do not obey proper scaling rules ${ }^{50-52}$ and

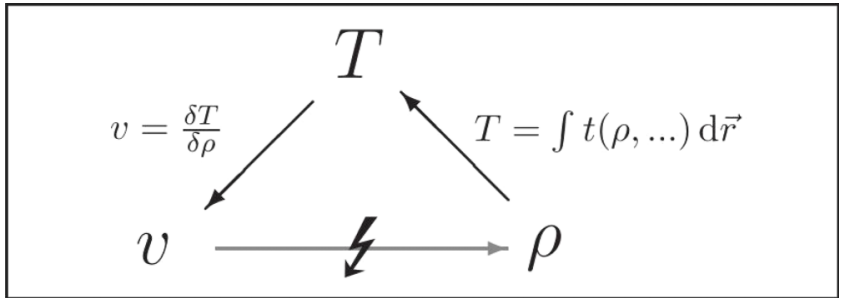

FIG. 10. Conventional approach for the design of kinetic energy density functionals.

consequently the virial theorem will be violated, ${ }^{53}$ leading to conceptual problems if one wishes to work within a consistent theoretical framework of density functional theory. ${ }^{11}$

Fig. 10 sketches the conventional route for the design of kinetic energy functionals. Usually, one starts constructing the kinetic energy in the form $T=\int t(\rho, \ldots) \mathrm{d} \vec{r}$, searching for such expressions that yield the KS energy if the KS density is inserted in the expression for the kinetic energy density. The fact that the kinetic energy is given as an integral over the kinetic energy density provides a straightforward route for obtaining the potential $v=\delta T[\rho] / \delta \rho$ as an analytic expression in terms of the density. Finally, this expression for the potential shall yield the right electron density by variational procedure. This is a closed and fully consistent circle within the framework of density functional theory, ${ }^{11}$ albeit the fact that the analytic expression for $T$ and $v$ is unknown. In practice, however, a consistent closed circle for atomistic systems with arbitrary number of electrons has not been found with a given functional expression for the kinetic energy in terms of the corresponding energy density despite huge efforts undertaken. ${ }^{5}$ Analytic expressions starting $\rho \mapsto t(\rho) \mapsto v(\rho)$ fail yielding back the proper density $v(\rho) \mapsto \rho$ s.

On the other hand, the current study and previous work on shell-structure-based approaches ${ }^{32}$ show that it is possible to obtain variational densities exhibiting proper atomic shell structure. Therefore, it seems possible to construct a potential that yields the exact electron density (or at least a reasonable approximation to it), see the sketch in Fig. 11. Moreover, from that electron density the proper kinetic energy of the system can be obtained, since the potential energy is known in terms of the density (at least in good approximation). By using the virial theorem, the kinetic and the total energy can be evaluated from the potential part, respectively. However, this procedure does not provide a closed and consistent circle within the framework of density functional theory. In case that the kinetic energy is obtained via the virial theorem, the potential can no more be considered to be the functional

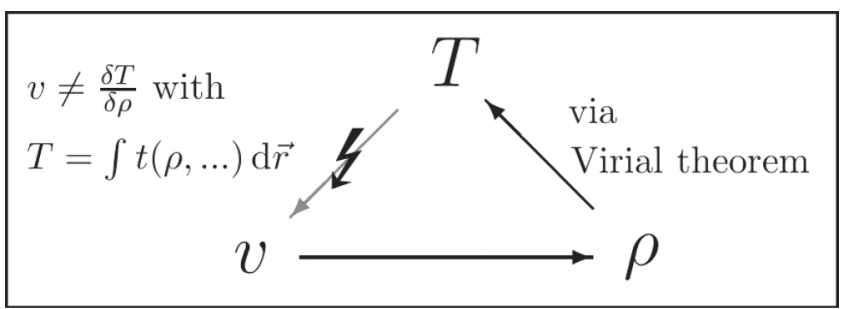

FIG. 11. Current approach approximating the potential. 
derivative of the energy, due to the break-down of the scaling rules. One can of course take the viewpoint of a closed circle and construct the corresponding $t(\rho)$ for the given potential Ansatz. Due to the wrong scaling behavior, the virial theorem will not be fulfilled and the resulting kinetic energy might be far away from the exact energy. In this viewpoint, the closure of the circle is maintained for the price of obtaining a bad energy estimate and the violation of the scaling rules. The requirement of a closed circle is of course a reasonable standpoint, since the exact Hohenberg-Kohn functional ${ }^{11}$ provides a closed and consistent circle. On the other hand, one can also adopt the viewpoint of a broken circle, meaning that one accepts to work with a non-consistent theoretical framework. In that case, one considers the analytic form for the potential $v$ as a given expression that yields the right electron density but is not the functional derivative of the kinetic energy at least not in the form $T=\int t(\rho, \ldots) \mathrm{d} \vec{r}$. Accepting that viewpoint the analytic expression for $v$ does not have to obey any scaling rule. This does not mean that $v$ itself does not respect the scaling rules, if one could find the "perfect" description, the scaling rules will of course be fulfilled since the KS counterparts obey those rules. But the chosen analytic approximation for $v$ is free of such requirements by taking such a viewpoint. This broken, non-consistent framework allows to treat the potential by a simple analytical expression. Moreover, that potential approximation yields an electron density with proper atomic shell structure by variational procedure. From that electron density a good estimate for the energy can be obtained by exploring the virial theorem.

Of course it would be desirable to obtain the above results within a consistent closed framework, but up to the authors knowledge no such expression is available for isolated systems.

Second, the issue of non-universality shall be addressed here. All SSB approaches ${ }^{31,32}$ are non-universal in the sense that they cannot be applied to model systems like the homogeneous electron gas. This is due to the fact that all SSB approaches live from the assumption that the atomic shell structure shall be incorporated in the functional expression (or the potential), which is an effect that is inherently related to the Coulombic nature of the system. However, the local conditions for the Pauli potential that have been worked out in this study are universal in the sense that they apply to all atoms in their ground state. In order to display the correct shell structure behavior of the electron density, the Pauli potential must be constructed in such a way that the resulting term $q$, cf., Eq. (9), exhibits $(2 n-1)$ roots, with $n$ being the row of the periodic table of the corresponding element. Of course the number of roots changes for elements standing in different rows, but the requirement stays the same. In that sense the local requirement is universal for all atoms. The same applies to the form of the Pauli potential. All second row elements (or ions with the corresponding electron number) can be described by the analytic form of the Pauli potential given by Eq. (14) or (17), since (like the local conditions for $q$ ) the form of the Pauli potential changes only if $n$ changes, see, e.g., Pauli potentials in Refs. 25 and 54.

Nevertheless, the proposed approximation for the Pauli potential is considered to be non-universal even for atoms with respect to the parameters that are used in the analytic expression (what makes a straightforward application to molecules or solids difficult), unless a unified description for those parameters can be found. Therefore, the aim is to search for a model that expresses the dependence of the parameters as a function of the nuclear charge in order to avoid a tedious parameterization for each atom separately. The author is not aware of any such model for the parameters used in Eq. (17), since such a model should provide the value of the electron density at the ideal shell positions. ${ }^{35}$ In that case the only possibility at current time is to take the values from the $\mathrm{Ne}$ potential, if one wishes to avoid reparametrization. Self-consistent electron densities for $\mathrm{Na}^{+}$and $\mathrm{F}^{-}$using the $\mathrm{Ne}$ potential are depicted in Figs. 12 and 13, respectively. The energetic data for a few selected ions from the isoelectronic series of $\mathrm{Ne}$ are compiled in Table II. As can be seen from the data the relative errors for the kinetic energy rapidly increase, by changing $Z$ while keeping the parameter values for the Pauli potential unchanged, cf., Eq. (17).

In contrast, the parameterization of Eq. (14) is straightforward. The best description of the Pauli potential

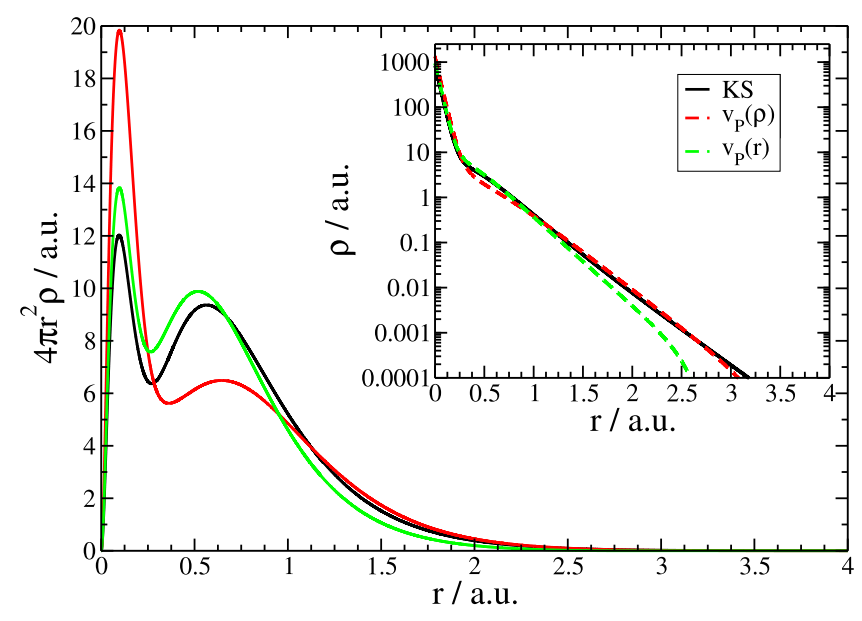

FIG. 12. Self-consistent electron densities for $\mathrm{Na}^{+}$obtained from orbital-free calculation with the Pauli potential according to Eqs. (14) and (17), respectively, in comparison with the KS electron density.

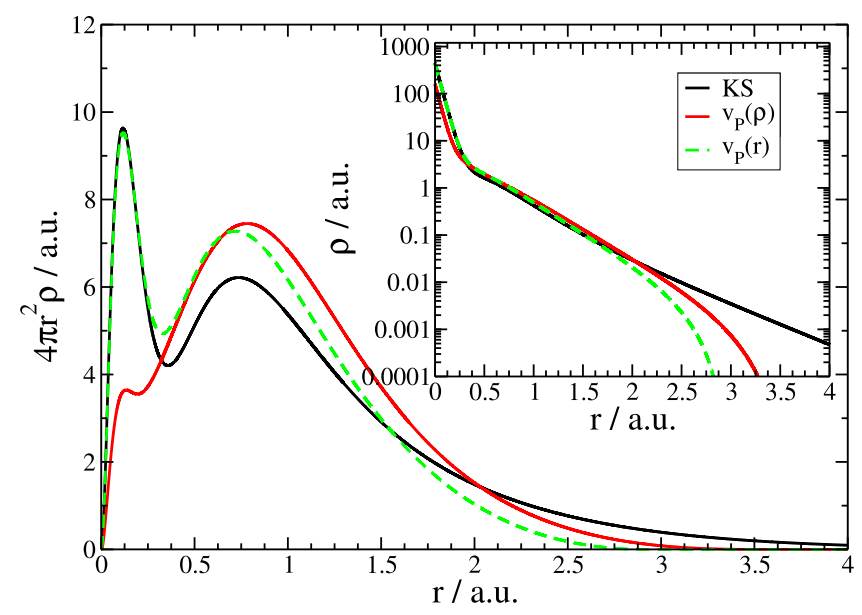

FIG. 13. Self-consistent electron densities for $\mathrm{F}^{-}$obtained from orbital-free calculation with the Pauli potential according to Eqs. (14) and (17), respectively, in comparison with the $\mathrm{KS}$ electron density. 
TABLE II. Potential and kinetic energies for the isoelectronic series of Ne. In case of KS calculations, $T$ has been evaluated as integral over the positive kinetic energy density and $V$ by using the virial theorem. In case of the OF DFT calculations, $V$ and $T_{\mathrm{W}}$ have been evaluated directly by integrating the corresponding energy densities, respectively. The total kinetic energy $T$ has been obtained by applying the virial theorem. Orbital-free calculations have been obtained with $\lambda=20$. All values are given in atomic units. ${ }^{53}$

\begin{tabular}{lccccccc}
\hline \hline $\mathrm{Z}$ & Method & $\mu$ & $V$ & $T$ & $T_{\mathrm{W}}$ & $T_{\mathrm{P}}$ & $\Delta T_{\text {rel }}$ \\
\hline 9 & $\mathrm{KS}$ & +0.12 & -197.3 & 98.6 & 71.7 & 27.0 & \\
& $v_{\mathrm{P}}(\rho)$ & -0.11 & -122.6 & 61.3 & 26.9 & 34.4 & $38 \%$ \\
& $v_{\mathrm{P}}(r)$ & +0.01 & -199.5 & 99.7 & 70.9 & 28.8 & $1 \%$ \\
10 & $\mathrm{KS}$ & -0.44 & -255.0 & 127.5 & 89.4 & 38.1 & \\
& $v_{\mathrm{P}}(\rho)$ & -0.75 & -251.9 & 126.0 & 89.7 & 36.3 & $1 \%$ \\
& $v_{\mathrm{P}}(r)$ & -0.89 & -270.9 & 135.4 & 95.8 & 39.7 & $6 \%$ \\
11 & $\mathrm{KS}$ & -1.28 & -320.9 & 160.5 & 109.2 & 51.2 & \\
& $v_{\mathrm{P}}(\rho)$ & -1.59 & -446.3 & 223.1 & 192.1 & 31.1 & $39 \%$ \\
& $v_{\mathrm{P}}(r)$ & -2.10 & -353.3 & 176.6 & 124.8 & 51.8 & $21 \%$ \\
12 & $\mathrm{KS}$ & -2.39 & -395.0 & 197.5 & 131.2 & 66.2 & \\
& $v_{\mathrm{P}}(\rho)$ & -2.63 & -675.8 & 337.9 & 311.8 & 26.1 & $71 \%$ \\
& $v_{\mathrm{P}}(r)$ & -3.51 & -450.9 & 225.4 & 159.7 & 65.7 & $14 \%$ \\
13 & $\mathrm{KS}$ & -3.75 & -475.0 & 237.5 & 154.4 & 82.0 & \\
& $v_{\mathrm{P}}(r)$ & -5.23 & -562.9 & 281.5 & 200.4 & 81.1 & $19 \%$ \\
& $\mathrm{KS}$ & -5.38 & -567.1 & 283.5 & 181.8 & 101.8 & \\
& $v_{\mathrm{P}}(r)$ & -7.25 & -690.1 & 345.0 & 247.1 & 97.9 & $22 \%$ \\
\hline \hline
\end{tabular}

has been obtained with $\alpha=4, \beta=8$. Therefore, the values for the exponents are kept fixed for all atoms. The parameter $b$ correlates with the eigenvalue difference between the first and the second shell. Obtaining this eigenvalue difference from the one-electron Schrödinger equation gives a rough estimate for that value. Since this model neglects electron-electron repulsion completely, the $b$ value was rescaled by $c_{b}=0.7733$ in case of the $\mathrm{Ne}$ atom. Although this scaling factor is slightly $Z$ dependent, it can be considered fixed in first approximation. This method yields the parameter $b=c_{b} * 3 / 8 * Z^{2}$ as a function of $Z$ for all atoms. The $b$ values have been calculated for the isoelectronic series of the $\mathrm{Ne}$ atom. The data are depicted by colored dots at the position $r=0$ bohr in Fig. 14

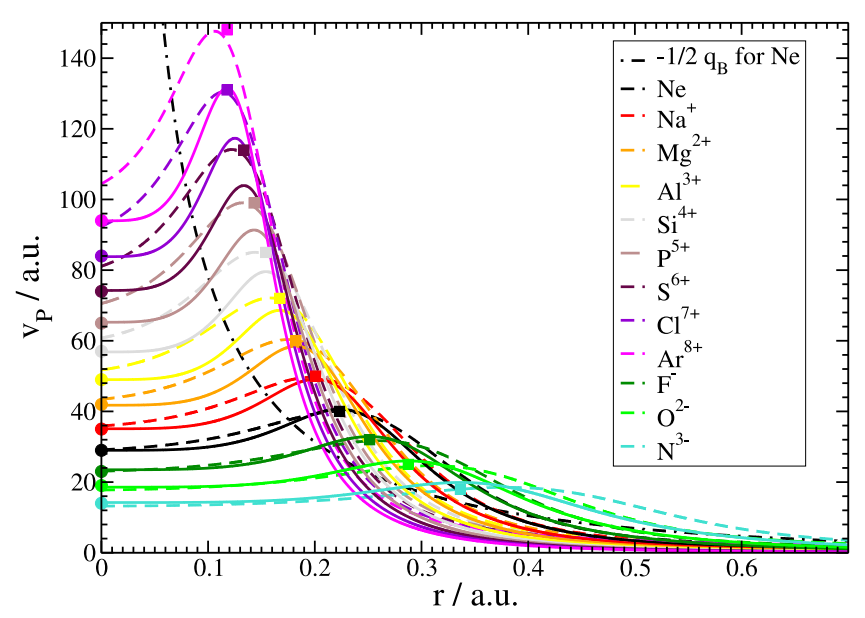

FIG. 14. Pauli potential for the isoelectronic series of Ne. Dashed lines: Pauli potential evaluated from KS orbitals, solid lines: SSB approximation according to Eq. (14). together with the Pauli potential obtained from KS orbitals, shown in the same color code. Next, $a$ and $c$ have to be determined. Since the maximum of the Pauli potential shall be located at the shell boundary, this results in determining $r_{1}$ and $\kappa$. The shell boundary $r_{1}$ is in good approximation known as a function of $Z$, namely, $r_{1}=\ln (Z /(Z-2)) .^{48}$ Those values are shown as colored squares $\left(r_{1}, y\right)$ in Fig. 14, whereby the $y$ value has been chosen to approximately match the maximum of the potential for pictorial convenience (the $y$ value is not needed in the calculation of the parameters). Given that $r_{1}$ is determined, $\kappa_{0}$ can be calculated. Recall that $\kappa_{0}$ is the minimum value in order to maintain the desired form of the potential. As long as $\kappa$ is chosen above $\kappa_{0}$, the form of the potential is not significantly influenced by $\kappa$. Therefore, $\kappa=1.4 \kappa_{0}$ is a good estimate that is kept fixed for all atoms.

Fig. 14 depicts the resulting approximations for the Pauli potential for the isoelectronic series of $\mathrm{Ne}$, with the above mentioned $Z$ dependence. The resulting self-consistent electron densities for $\mathrm{Na}^{+}$and $\mathrm{F}^{-}$are shown in Figs. 12 and 13 , respectively. The corresponding energetic data are compiled in Table II. As can be seen from the results, the analytic form of the Pauli potential as given by Eq. (14) or (17) is transferable within an isoelectronic series. The resulting self-consistent electron densities will all exhibit the proper atomic shell structure. This fact can be deduced from Fig. 14, where the various potential approximations are plotted for the isoelectronic series of Ne together with $-1 / 2 q_{\mathrm{B}}$ for the $\mathrm{Ne}$ atom. The latter serves as a rough estimate for the corresponding function for different $Z$, showing that the number of roots for $q$ is preserved in all cases. The quality of the orbital-free electron density with respect to the values does of course decrease by changing the nuclear charge if the potential is taken from the Ne example, but a reparametrization of Eq. (14) is straightforward, significantly ameliorating the results, see also data in Table II.

\section{CONCLUSIONS}

In the present study, the local conditions for the Pauli potential necessary in order to yield self-consistent electron densities from orbital-free calculations were determined for potentials given in terms of a local position variable and additionally, for approximations which are given in terms of the electron density. For atoms in their ground state, the Pauli potential must be constructed in such a way that the resulting function $q$, determining the radial electron density, exhibits $(2 n-1)$ roots, whereby $n$ is the row of the corresponding element in the periodic table. All approximations for the Pauli potential respecting this local requirement yield self-consistent electron densities with proper shell structure, due to the fact that the number of roots for $q$ is preserved in each iteration step.

Based on those findings, an explicit formula for the Pauli potential in terms of $\rho$ was proposed for the $\mathrm{Ne}$ atom. This explicit expression preserves the local requirements for the Pauli potential during each iteration step, and consequently, the resulting electron density displays the proper atomic shell structure for the $\mathrm{Ne}$ atom. Therefore, this study demonstrates that it is possible to find simple local one-point approximations 
for the Pauli potential given in terms of the electron density, that are able to yield self-consistent electron densities with proper atomic shell structure via Hohenberg-Kohn variational principle.

\section{ACKNOWLEDGMENTS}

The author wishes to express her thanks to Kieron Burke for fruitful discussions about the virial theorem and to Frank R. Wagner for technical help with the ADF calculations. The Alexander von Humboldt foundation is greatly acknowledged for funding this work.

${ }^{1}$ Y. A. Wang and E. A. Carter, in Theoretical Methods in Condensed Phase Chemistry, edited by S. D. Schwarz (Kluwer, New York, 2000), pp. 117-184.

${ }^{2}$ V. Karasiev, R. S. Jones, S. B. Trickey, and F. E. Harris, in New Developments in Quantum Chemistry, edited by E. J. Paz and A. J. Hernandez (Transworld Research Network, Trivandrum, 2009).

${ }^{3}$ V. V. Karasiev and S. B. Trickey, Comput. Phys. Commun. 183, 2519 (2012).

${ }^{4}$ V. Karasiev, D. Chakraborty, and S. B. Trickey, in Many-Electron Approaches in Physics, Chemistry and Mathematics: A Multidisciplinary View, edited by L. Delle Site and V. Bach (Springer Verlag, 2013).

${ }^{5}$ V. Karasiev and S. B. Trickey, Adv. Quantum Chem. 71, 221-245 (2015).

${ }^{6}$ L. H. Thomas, Proc. Cambridge Philos. Soc. 23, 542 (1927).

${ }^{7}$ E. Fermi, Z. Phys. 48, 73 (1928).

${ }^{8}$ C. F. von Weizsäcker, Z. Phys. 96, 431 (1935).

${ }^{9}$ D. A. Kirzhnits, Sov. Phys. JETP 5, 64 (1957).

${ }^{10}$ C. H. Hodges, Can. J. Phys. 51, 1428 (1973).

${ }^{11}$ P. Hohenberg and W. Kohn, Phys. Rev. B 136, 864 (1964).

${ }^{12}$ W. Yang, Phys. Rev. A 34, 4575 (1986).

${ }^{13}$ A. E. DePristo and J. D. Kress, Phys. Rev. A 35, 438 (1987).

${ }^{14}$ A. J. Thakkar, Phys. Rev. A 46, 6920 (1992).

${ }^{15}$ D. J. Lacks and R. G. Gordon, J. Chem. Phys. 100, 4446 (1994).

${ }^{16}$ F. Tran and T. A. Wesolowski, Int. J. Quantum Chem. 89, 441 (2002).

${ }^{17}$ L. M. Ghiringhelli and L. Delle Site, Phys. Rev. B 77, 073104 (2008).

${ }^{18}$ L. M. Ghiringhelli, I. P. Hamilton, and L. Delle Site, J. Chem. Phys. 132, 014106 (2010).

${ }^{19}$ S. Trickey, V. V. Karasiev, and A. Vela, Phys. Rev. B 84, 075146 (2011).

${ }^{20}$ I. Shin and E. A. Carter, J. Chem. Phys. 140, 18A531 (2014).
${ }^{21}$ N. H. March, Phys. Lett. A 113, 476 (1986).

${ }^{22}$ R. M. Dreizler and E. K. U. Gross, Density Functional Theory (Springer Verlang, Berlin Heidelberg, 1990).

${ }^{23}$ P. W. Ayers and S. Liu, Phys. Rev. A 75, 022514 (2007).

${ }^{24}$ E. V. Ludeña, F. Illas, and A. Ramirez-Solis, Int. J. Mod. Phys. B 22, 4642 (2008).

${ }^{25}$ A. Nagy, Acta Phys. Hung. 70, 321 (1991).

${ }^{26}$ A. Nagy and N. H. March, Int. J. Quantum Chem. 39, 615 (1991).

${ }^{27}$ A. Nagy and N. H. March, Phys. Chem. Liq. 25, 37 (1992).

${ }^{28}$ A. Nagy, Chem. Phys. Lett. 460, 343 (2008).

${ }^{29}$ A. Nagy, Int. J. Quantum Chem. 110, 2117 (2010).

${ }^{30}$ A. Nagy, J. Chem. Phys. 135, 044106 (2011).

${ }^{31}$ K. Finzel, Theor. Chem. Acc. 134, 106 (2015).

${ }^{32}$ K. Finzel, Int. J. Quantum Chem. 115, 1629 (2015).

${ }^{33}$ L. S. Bartell and L. O. Brockway, Phys. Rev. 90, 833 (1953).

${ }^{34}$ J. T. Waber and D. T. Cromer, J. Chem. Phys. 42, 4116 (1965).

${ }^{35}$ H. Schmider, R. Sagar, and V. H. Smith, Jr., Can. J. Chem. 70, 506 (1992).

${ }^{36}$ H. Schmider, R. Sagar, and V. H. Smith, Jr., Proc. Indiana Acad. Sci. 106, 133 (1994).

${ }^{37}$ M. Kohout, A. Savin, and H. Preuss, J. Chem. Phys. 95, 1928 (1991).

${ }^{38}$ A. Savin, O. Jepsen, J. Flad, O. K. Anderson, H. Preuss, and H. G. von Schnering, Angew. Chem., Int. Ed. 31, 187 (1992).

${ }^{39}$ M. Kohout and A. Savin, Int. J. Quantum Chem. 60, 875 (1996).

${ }^{40}$ K. Wagner and M. Kohout, Theor. Chem. Acc. 128, 39 (2011).

${ }^{41}$ K. Finzel, Y. Grin, and M. Kohout, Theor. Chem. Acc. 131, 1106 (2012).

${ }^{42}$ K. Finzel and M. Kohout, Theor. Chem. Acc. 132, 1392 (2013).

${ }^{43}$ K. Finzel, Int. J. Quantum Chem. 114, 1546 (2014).

${ }^{44}$ M. Levy, J. P. Perdew, and V. Sahni, Phys. Rev. A 30, 2745 (1984).

${ }^{45} \mathrm{SCM}$, Theoretical Chemistry, Vrije Universiteit, Amsterdam, The Netherlands, ADF2012.01, 2012, http://www.scm.com.

${ }^{46}$ C. Zener, Phys. Rev. 36, 51 (1930).

${ }^{47}$ J. C. Slater, Phys. Rev. 36, 57 (1930).

${ }^{48} \mathrm{~K}$. Finzel, "Reinvestigation of the ideal atomic shell structure and its application in orbital-free density functional theory" (submitted).

${ }^{49}$ J. P. Perdew, A. Ruzsinszky, J. Sun, and K. Burke, J. Chem. Phys. 140, 18A533 (2014).

${ }^{50}$ M. Levy and J. P. Perdew, Phys. Rev. A 32, 2010 (1985).

${ }^{51}$ M. Levy and H. Ou-Yang, Phys. Rev. A 38, 625 (1988).

${ }^{52}$ H. Ou-Yang and M. Levy, Phys. Rev. A 42, 155 (1990).

${ }^{53}$ G. R. Parr and W. Yang, Density-Functional Theory of Atoms and Molecules (Oxford University Press, 1989).

${ }^{54}$ Q. Zhao and R. G. Parr, Phys. Rev. A 46, 2337 (1992). 